\title{
Order Theoretic Common n-tuple Fixed Point
}

\author{
Yaé Ulrich Gaba
}

University of Cape Town, Rondebosch 7701, South Africa.

\begin{abstract}
In this article, we solve an open problem initially suggested in [2], namely:
Let $(\mathrm{X}, \mathrm{d})$ be a Hausdorff left $\mathrm{K}$-complete $T_{0}$-quasi-pseudometric space, $\phi: X \rightarrow \mathbb{R}$ be a bounded from above function and $\preceq$ the preorder induced by $\phi$. Let $F: X \times X \rightarrow X$ and $G_{i}: X \rightarrow$ $X ; i=1,2, \cdots, N$ for $N>2$ be $N+1 d$-sequentially continuous mapping on $X$ such that the pairs $\left\{F ; G_{i}\right\} ; i=1,2, \cdots, N$ are weakly left-related.
\end{abstract}

Problem:

1. Can we prove that $F, G_{1}, \cdots, G_{N}$ have a common coupled fixed point in $X$ ?

2. Alternatively, what could be a correct formulation of the statement, using the induced preorder and the weakly left-related property that guarantees a positive answer?

We answer this question by the affirmative. In fact we prove that a more general result holds when $F: X^{n} \rightarrow X$ for a natural number $n>2$.

Keywords: Quasi-pseudometric space, left $K$-complete, preordered space, left-weakly related, common couple fixed point, common n-tuple fixed

\section{Preliminaries}

Definition 1.1. Let $\left(X, \preceq_{X}\right)$ and $\left(Y, \preceq_{Y}\right)$ be two prosets. A map $T: X \rightarrow Y$ is said to be preorderpreserving or isotone if for any $x, y \in X$,

$$
x \preceq_{X} y \Longrightarrow T x \preceq_{Y} T y .
$$

Similarly, for any family $\left(X_{i}, \preceq_{X_{i}}\right), i=1,2, \cdots, n ;\left(Y, \preceq_{Y}\right)$ of posets, a mapping $F: X_{1} \times X_{2} \times \cdots \times X_{n} \rightarrow$ $Y$ is said to be preorder-preserving or isotone if for any for any $\left(x_{1}, x_{2}, \cdots, x_{n}\right),\left(z_{1}, z_{2}, \cdots, z_{n}\right) \in$ $X_{1} \times X_{2} \times \cdots \times X_{n}$

$$
x_{i} \preceq_{X_{i}} z_{i} \text { for all } i=1,2, \cdots, n \Longrightarrow F\left(x_{1}, x_{2}, \cdots, x_{n}\right) \preceq_{Y} F\left(z_{1}, z_{2}, \cdots, z_{n}\right) .
$$

Definition 1.2. (Compare [4]) Let $X$ be a non empty set. A function $d: X \times X \rightarrow[0, \infty)$ is called a quasi-pseudometric on $X$ if:

i) $d(x, x)=0 \quad \forall x \in X$

ii) $d(x, z) \leq d(x, y)+d(y, z) \quad \forall x, y, z \in X$.

Moreover, if $d(x, y)=0=d(y, x) \Longrightarrow x=y$, then $d$ is said to be a $T_{0}$-quasi-pseudometric. The latter condition is referred to as the $T_{0}$-condition.

\section{Remark 1.3.}

- Let $d$ be a quasi-pseudometric on $X$, then the map $d^{-1}$ defined by $d^{-1}(x, y)=d(y, x)$ whenever $x, y \in X$ is also a quasi-pseudometric on $X$, called the conjugate of $d$. In the literature, $d^{-1}$ is also denoted $d^{t}$ or $\bar{d}$.

- It is easy to verify that the function $d^{s}$ defined by $d^{s}:=d \vee d^{-1}$, i.e. $d^{s}(x, y)=\max \{d(x, y), d(y, x)\}$ defines a metric on $X$ whenever $d$ is a $T_{0}$-quasi-pseudometric on $X$. 
Definition 1.4. Let $(X, d)$ be a quasi-pseudometric space. The convergence of a sequence $\left(x_{n}\right)$ to $x$ with respect to $\tau(d)$, called d-convergence or left-convergence and denoted by $x_{n} \stackrel{d}{\longrightarrow} x$, is defined in the following way

$$
x_{n} \stackrel{d}{\longrightarrow} x \Longleftrightarrow d\left(x, x_{n}\right) \longrightarrow 0 .
$$

Finally, in a quasi-pseudometric space $(X, d)$, we shall say that a sequence $\left(x_{n}\right) d^{s}$-converges to $x$ if it is both left and right convergent to $x$, and we denote it as $x_{n} \stackrel{d^{s}}{\longrightarrow} x$ or $x_{n} \longrightarrow x$ when there is no confusion. Hence

$$
x_{n} \stackrel{d^{s}}{\longrightarrow} x \Longleftrightarrow x_{n} \stackrel{d}{\longrightarrow} x \text { and } x_{n} \stackrel{d^{-1}}{\longrightarrow} x .
$$

Definition 1.5. A sequence $\left(x_{n}\right)$ in a quasi-pseudometric $(X, d)$ is called

(a) left $d$-Cauchy if for every $\epsilon>0$, there exist $x \in X$ and $n_{0} \in \mathbb{N}$ such that

$$
\forall n \geq n_{0}, \quad d\left(x, x_{n}\right)<\epsilon ;
$$

(b) left $K$-Cauchy if for every $\epsilon>0$, there exists $n_{0} \in \mathbb{N}$ such that

$$
\forall n, k: n_{0} \leq k \leq n, \quad d\left(x_{k}, x_{n}\right)<\epsilon ;
$$

(c) $d^{s}$-Cauchy if for every $\epsilon>0$, there exists $n_{0} \in \mathbb{N}$ such that

$$
\forall n, k \geq n_{0}, \quad d\left(x_{n}, x_{k}\right)<\epsilon
$$

Definition 1.6. (Compare [4]) A quasi-pseudometric space $(X, d)$ is called

- left-K-complete provided that any left $K$-Cauchy sequence is d-convergent,

- left Smyth sequentially complete if any left $K$-Cauchy sequence is $d^{s}$-convergent.

Definition 1.7. A $T_{0}$-quasi-pseudometric space $(X, d)$ is called bicomplete provided that the metric $d^{s}$ on $X$ is complete.

Definition 1.8. Let $(X, d)$ be a quasi-pseudometric type space. A function $T: X \rightarrow X$ is called $d$ sequentially continuous or left-sequentially continuous if for any d-convergent sequence $\left(x_{n}\right)$ with $x_{n} \stackrel{d}{\longrightarrow} x$, the sequence $\left(T x_{n}\right) d$-converges to $T x$, i.e. $T x_{n} \stackrel{d}{\longrightarrow} T x$.

Similarly, a function $T: X_{1} \times X_{2} \times \cdots \times X_{n} \rightarrow X$ for $n \geq 2$, is said to be $d$-sequentially continuous or left-sequentially continuous if for any sequences $\left(x_{l}^{i}\right)$ such that $x_{l}^{i} \stackrel{d}{\longrightarrow} x^{*, i}$, then

$$
T\left(x_{l}^{i}, x_{l}^{i+1}, \cdots, x_{l}^{n}, x_{l}^{i}, \cdots, x_{l}^{i-1}\right) \stackrel{d}{\longrightarrow} T\left(x^{*, i}, x^{*, i+1}, \cdots, x^{*, n}, x^{*, 1}, \cdots, x^{*, i-1}\right) .
$$

Definition 1.9. (Compare [1]) An element $\left(x^{1}, x^{2}, \cdots, x^{n}\right) \in X^{n}$ is called:

(E1) a n-tuple fixed point of the mapping $F: X^{n} \rightarrow X$ if

$$
F\left(x^{i}, x^{i+1}, \cdots, x^{n}, x^{1}, \cdots, x^{i-1}\right)=x^{i}, \text { for all } i, 1 \leq i \leq n .
$$

(E2) a n-tuple coincidence point of the mappings $F: X^{n} \rightarrow X$ and $T: X \rightarrow X$ if

$$
F\left(x^{i}, x^{i+1}, \cdots, x^{n}, x^{1}, \cdots, x^{i-1}\right)=T x^{i}
$$

for all $i, 1 \leq i \leq n$ and in this case $\left(T x^{1}, T x^{2}, \cdots, T x^{n}\right)$ is called the $n$-tuple point of coincidence; (E3) a common n-tuple fixed point of the mappings $F: X^{n} \rightarrow X$ and $T: X \rightarrow X$ if

$$
F\left(x^{i}, x^{i+1}, \cdots, x^{n}, x^{1}, \cdots, x^{i-1}\right)=T x^{i}=x^{i}
$$

for all $i, 1 \leq i \leq n$. 
From the above, we then obtained the natural following definitions

Definition 1.10. Let $X$ be a non empty set. An element $\left(x^{1}, x^{2}, \cdots, x^{n}\right) \in X^{n}$ is called:

(E'Q) a n-tuple coincidence point of the mappings $F: X^{n} \rightarrow X$ and $G_{k}: X \rightarrow X$ with $k, 1 \leq k \leq$ $N, N>2$ if $F\left(x^{i}, x^{i+1}, \cdots, x^{n}, x^{1}, \cdots, x^{i-1}\right)=G_{k} x^{i}$ for all $i, 1 \leq i \leq n$ and for all $k, 1 \leq k \leq N$;

(E'2) a n-tuple common fixed point of the mappings $F: X^{n} \rightarrow X$ and $G_{1}, G_{2}, \cdots, G_{N}: X \rightarrow X$ with $N>2$ if $F\left(x^{i}, x^{i+1}, \cdots, x^{n}, x^{1}, \cdots, x^{i-1}\right)=G_{k} x^{i}=x^{i}$ for all $i, 1 \leq i \leq n$ and for all $k, 1 \leq k \leq N$.

Definition 1.11. (See [2]) Let $(X, \preceq)$ be a preordered space, and $F: X^{n} \rightarrow X$ and $g: X \rightarrow X$ be two mappings. Then the pair $\{F, g\}$ is said to be weakly left-related if the following condition is satisfied: and

$$
F\left(x^{i}, x^{i+1}, \cdots, x^{n}, x^{1}, \cdots, x^{i-1}\right) \preceq g F\left(x^{i}, x^{i+1}, \cdots, x^{n}, x^{1}, \cdots, x^{i-1}\right)
$$

$$
g x^{i} \preceq F\left(g x^{i}, g x^{i+1}, \cdots, g x^{n}, g x^{1}, \cdots, x^{i-1}\right)
$$

for all $1 \leq i \leq n$.

\section{Main Result}

We recall the following lemma.

Lemma 2.1. (Compare [2]) Let $(X, d)$ be a quasi-pseudometric space and $\phi: X \rightarrow \mathbb{R}$ a map. Define the binary relation " $\preceq "$ on $X$ as follows:

$$
x \preceq y \Longleftrightarrow d(x, y) \leq \phi(y)-\phi(x) .
$$

Then " $\preceq "$ is a preorder on $X$. It will be called the preorder induced by $\phi$.

Example 2.2. Let $X=[0, \infty)$ and $d(x, y)=\max \{0, x-y\}$. Then $(X, d)$ is a quasi-pseudometric space. For any positive real number $t$, let $\phi_{t}: X \rightarrow \mathbb{R}$ defined by $\phi_{t}(x)=t x$. Then for $x, y \in X$, we have

$$
\begin{aligned}
x \preceq y & \Longleftrightarrow d(x, y) \leq \phi_{t}(y)-\phi_{t}(x) \\
& \Longleftrightarrow 0=\max \{0, x-y\} \leq t(y-x) .
\end{aligned}
$$

It follows that $3 / 2 \preceq 3,1 / 2 \preceq 1$, etc.

Remark 2.3. (Compare [2]) If in addition, the space $(X, d)$ is $T_{0}$, then the relation $\preceq$ defined by

$$
x \preceq y \Longleftrightarrow d^{s}(x, y) \leq \phi(y)-\phi(x) .
$$

is a partial order on $X$.

We introduce the following definition.

Definition 2.4. Let $(X, \preceq)$ be a preordered set and $g, f: X \rightarrow X$. We say that the pair $\{g, f\}$ (in this order) is an embedded pair if

$$
g(x) \preceq f(g(x)), \text { whenever } x \in X .
$$

We shall say that the family $\left\{G_{1}, G_{2}, \cdots, G_{n}\right\}$ (in this order) is a n-embedded chain if for all $i=$ $1, \cdots, n-1$, the pair $\left\{G_{i}, G_{i+1}\right\}$ is an embedded pair. Observe that an embedded pair is a 2-embedded chain.

We shall say that the family $\left\{G_{1}, G_{2}, \cdots, G_{n}\right\}$ is a dual n-embedded chain if $\left\{G_{1}, G_{2}, \cdots, G_{n}\right\}$ and $\left\{G_{n}, G_{n-1}, \cdots, G_{1}\right\}$ are $n$-embedded chains. 
Example 2.5. Let $X=[2, \pi)$ with the usual order and consider the pairs $\mathcal{F}=\left\{F_{1}(x)=3 x, F_{2}(x)=5 x\right\}$ and $\mathcal{G}=\left\{G_{1}(x)=\sin x+1, G_{2}(x)=x^{2}\right\}$.

For any $x \in X$,

$$
F_{1}(x)=3 x \leq 5(3 x)=F_{2}\left(F_{1}(x)\right) \text { and } F_{2}(x)=5 x \leq 3(5 x)=F_{1}\left(F_{2}(x)\right),
$$

showing that $\mathcal{F}$ is a dual 2-embedded chain.

On the other way around

$$
x \in X, G_{1}(x)=\sin x+1 \leq(\sin x+1)^{2}=G_{2}\left(G_{1}(x)\right),
$$

showing that $\mathcal{G}$ is an embedded pair, while

$$
G_{2}(x)=x^{2}>\sin \left(x^{2}\right)+1=G_{1}\left(G_{2}(x)\right),
$$

showing that $\mathcal{G}$ is not a dual 2-embedded chain.

Now we are ready to give the solution to the open problem.

Theorem 2.6. Let $(Y, d)$ be a Hausdorff left $K$-complete $T_{0}$-quasi-pseudometric space, $\phi: Y \rightarrow \mathbb{R}$ be a bounded from above function and $\preceq$ the preorder induced by $\phi$. Let $F: Y \times Y \rightarrow Y$ and $G_{i}: Y \rightarrow Y ; i=$ $2, \cdots, r$ for $r>2$ be $(r-1)+1 d$-sequentially continuous mapping on $X$ such that the pairs $\left\{F, G_{r}\right\} ; r=2,3$ are weakly left-related. Moreover, we assume that $\left\{G_{r}, G_{r-1}, \cdots, G_{3}\right\}$ is an $r-2$-embedded chain. Then $F, G_{2}, \cdots, G_{r}$ have a common n-tuple fixed point in $Y$.

Proof. Let $X_{0}^{1}, \cdots, X_{0}^{n} \in X$. We construct the sequences $\left(X_{l}^{i}\right)_{l}$ in $Y$ as follows:

$$
G_{r} X_{r l-r}^{i}=X_{r l-r+1}^{i}, \cdots, G_{3} X_{r l-3}^{i}=X_{r l-2}^{i}, G_{2} X_{r l-1}^{i}=X_{r l}^{i}
$$

and

$$
X_{r l-1}^{i}=F\left(X_{r l-2}^{i}, X_{r l-2}^{i+1}, \cdots, X_{r l-2}^{n}, X_{r l-2}^{1}, \cdots, X_{r l-2}^{i-1}\right),
$$

for all $l \geq 1$. We shall show that

$$
X_{l}^{i} \preceq X_{l+1}^{i} \quad \text { for all } i, 1 \leq i \leq n .
$$

Since the pair $\left\{G_{r}, G_{r-1}\right\}$ is an embedded pair, we have

$$
X_{1}^{i}=G_{r} X_{0}^{i} \preceq G_{r-1}\left(G_{r} X_{0}^{i}\right)=G_{r-1}\left(X_{1}^{i}\right)=X_{2}^{i} .
$$

Again, since the pair $\left\{G_{r-1}, G_{r-2}\right\}$ is an embedded pair, we have

$$
X_{2}^{i}=G_{r-1} X_{1}^{i} \preceq G_{r-2}\left(G_{r-1} X_{1}^{i}\right)=G_{r-2}\left(X_{2}^{i}\right)=X_{3}^{i} .
$$

So we obtain recursively

$$
G_{r} X_{0}^{i}=X_{1}^{i} \preceq G_{r-1} X_{1}^{i}=X_{2}^{i} \preceq \cdots \preceq G_{3}\left(X_{r-3}^{i}\right)=X_{r-2}^{i} .
$$

Now, since the pair $\left\{F, G_{3}\right\}$ is weakly left-related, we have

$$
\begin{aligned}
X_{r-2}^{i}=G_{3}\left(X_{r-3}^{i}\right) & \preceq F\left(G_{3} X_{r-3}^{i}, G_{3} X_{r-3}^{i+1}, \cdots, G_{3} X_{r-3}^{n}, G_{3} X_{r-3}^{1}, \cdots, G_{3} X_{r-3}^{i-1}\right) \\
& =F\left(X_{r-2}^{i}, X_{r-2}^{i+1}, \cdots, X_{r-2}^{n}, X_{r-2}^{1}, \cdots, X_{r-2}^{i-1}\right)=X_{r-1}^{i} .
\end{aligned}
$$

Again since the pair $\left\{F, G_{2}\right\}$ is weakly left-related, we have

$$
\begin{aligned}
X_{r-1}^{i} & =F\left(X_{r-2}^{i}, X_{r-2}^{i+1}, \cdots, X_{r-2}^{n}, X_{r-2}^{1}, \cdots, X_{r-2}^{i-1}\right) \\
& \preceq G_{2} F\left(X_{r-2}^{i}, X_{r-2}^{i+1}, \cdots, X_{r-2}^{n}, X_{r-2}^{1}, \cdots, X_{r-2}^{i-1}\right) \\
& =G_{2} X_{r-1}^{i}=X_{r}^{i} .
\end{aligned}
$$


Similarly, using repeatedly the fact that the pairs $\left\{F, G_{2}\right\}$ and $\left\{F, G_{3}\right\}$ are weakly left-related, and that $\left\{G_{r}, G_{r-1}, \cdots, G_{3}\right\}$ is an an $r-2$-embedded chain, we obtain

$$
X_{1}^{i} \preceq X_{2}^{i} \preceq X_{3}^{i} \preceq \ldots \preceq X_{l}^{i} \preceq \cdots
$$

By definition of the preorder, we have

$$
\phi\left(X_{1}^{i}\right) \leq \phi\left(X_{2}^{i}\right) \leq \ldots \leq \phi\left(X_{l}^{i}\right) \leq \cdots,
$$

Hence, the sequence $\left(\phi\left(X_{l}^{i}\right)\right)$ is a non-decreasing sequence of real numbers. Since $\phi$ is bounded from above, the sequence $\left(\phi\left(X_{l}^{i}\right)\right)$ converges and is therefore Cauchy. This entails that for any $\varepsilon>0$, there exists $n_{0} \in \mathbb{N}$ such that for any $q>p>n_{0}$, we have $\phi\left(X_{q}^{i}\right)-\phi\left(X_{p}^{i}\right)<\varepsilon$. Since whenever $q>p>n_{0}, X_{p}^{i} \preceq X_{q}^{i}$ and it follows that

$$
d\left(X_{p}^{i}, X_{q}^{i}\right) \leq \phi\left(X_{q}^{i}\right)-\phi\left(X_{p}^{i}\right)<\varepsilon .
$$

We conclude that $\left(X_{l}^{i}\right)$ is a left $K$-Cauchy sequence in $Y$ and since $Y$ is left $K$-complete, there exist $X^{*, i} \in Y$ such that $X_{l}^{i} \stackrel{d}{\longrightarrow} X^{*, i}$.

Since $F$ and $G_{2}, \cdots, G_{r}$ are $d$-sequentially continuous, it is easy to see that

$$
\begin{aligned}
X_{r l-1}^{i} \stackrel{d}{\longrightarrow} X^{*, i} & \Longleftrightarrow F\left(X_{r l-2}^{i}, X_{r l-2}^{i+1}, \cdots, X_{r l-2}^{n}, X_{r l-2}^{1}, \cdots, X_{r l-2}^{i-1}\right) \stackrel{d}{\longrightarrow} X^{*, i} \\
& \Longleftrightarrow F\left(X^{*, i}, X^{*, i+1}, \cdots, X^{*, n}, X^{*, 1} \cdots, X^{*, i-1}\right)=X^{*, i}
\end{aligned}
$$

and

$$
X_{r l-r}^{i} \stackrel{d}{\longrightarrow} X^{*, i} \Longleftrightarrow X_{r l-k+1}^{i}=G_{k} X_{r l-k}^{i} \stackrel{d}{\longrightarrow} X^{*, i} \Longleftrightarrow G_{k} X^{*, i}=X^{*, i}
$$

and hence

$$
G_{k} X^{*, i}=X^{*, i}=F\left(X^{*, i}, X^{*, i+1}, \cdots, X^{*, n}, X^{*, 1} \cdots, X^{*, i-1}\right) .
$$

Hence $\left(X^{*, 1}, X^{*, 2}, \cdots, X^{*, n}\right)$ is a common n-tuple fixed point of $F$ and $G_{2}, \cdots, G_{r}$.

Example 2.7. Let $X=[0, \infty)$ and $d: X \times X \rightarrow \mathbb{R}$ be the mapping defined by $d(a, b)=\max \{a-b, 0\}$. Then $d$ is a $T_{0}$-quasi-pseudometric on $X$. Observe that any left $K$-Cauchy sequence in $(X, d)$ is $d$-convergent to 0 . Indeed, if $\left(x_{n}\right)$ is a left $K$-Cauchy sequence, for every $\epsilon>0$, there exists $n_{0} \in \mathbb{N}$ such that

$$
\forall n, k: n_{0} \leq k \leq n \quad d\left(x_{k}, x_{n}\right)<\epsilon .
$$

This entails that $\forall n: n_{0}<n$

$$
d\left(0, x_{n}\right) \leq d\left(0, x_{n-1}\right)+d\left(x_{n-1}, x_{n}\right)=0+d\left(x_{n-1}, x_{n}\right)<\epsilon .
$$

Hence $d\left(0, x_{n}\right) \longrightarrow 0$, i.e. $x_{n} \stackrel{d}{\longrightarrow} 0$. Therefore $(X, d)$ is left $K$-complete.

For any positive real number $a$, let $\phi_{a}: X \rightarrow \mathbb{R}$ be defined by $\phi_{a}(x)=$ ax, and $\preceq$ the preorder induced by $\phi_{a}$. We define $F: X^{n} \rightarrow X$ and $G X \rightarrow X$ as follows

$$
F\left(x^{1}, x^{2}, \cdots, x^{n}\right)=x^{1}+\left|\sin \left(x^{1} x^{2} \cdots x^{n}\right)\right| \text { and } G_{k}(x)=k x, k=2, \cdots, r, r>2 .
$$

For $k=1,2$, we have on one hand,

$$
G_{k} F\left(x^{i}, x^{i+1}, \cdots, x^{n}, x^{1}, \cdots, x^{i-1}\right)=k\left(x^{i}+\left|\sin \left(x^{1} x^{2} \cdots x^{n}\right)\right|\right),
$$

i.e.

$$
F\left(x^{i}, x^{i+1}, \cdots, x^{n}, x^{1}, \cdots, x^{i-1}\right) \preceq G_{k} F\left(x^{i}, x^{i+1}, \cdots, x^{n}, x^{1}, \cdots, x^{i-1}\right),
$$

and on the other hand, 


$$
\begin{aligned}
F\left(G_{k} x^{i}, G_{k} x^{i+1}, \cdots, G_{k} x^{n}, G_{k} x^{1}, \cdots, G_{k} x^{i-1}\right) & =F\left(k x^{i}, k x^{i+1}, \cdots, k x^{n}, k x^{1}, \cdots, k x^{i-1}\right) \\
& =k x^{i}+\left|\sin \left(k^{n} x^{1} x^{2} \cdots x^{n}\right)\right|
\end{aligned}
$$

i.e.

$$
G_{k} x^{i} \preceq F\left(G_{k} x^{i}, G_{k} x^{i+1}, \cdots, G_{k} x^{n}, G_{k} x^{1}, \cdots, G_{k} x^{i-1}\right) .
$$

And so the pair $\left\{F, G_{k}\right\}$ are weakly left-related for $k=2,3$. Again, it is not hard to see that $F$ and $G_{k}, k=2, \cdots, r$, are $d$-sequentially continuous mappings on $X$.

Moreover, for any $x \in[0, \infty), k x \leq k(k-1) x, k=2, \cdots, r$, implying that $\left\{G_{r}, G_{r-1}, \cdots, G_{3}\right\}$ is an an $r-2$-embedded chain. Hence we see that all the conditions of our theorem are satisfied.

Also we have

$$
F\left(0, x^{i}, x^{i+1}, \cdots, x^{n}, x^{1}, \cdots, x^{i-2}\right)=0=G_{k}(0)
$$

for $k=2, \cdots, r$ and for $i=1,2, \cdots, n$.

Thus $\underbrace{(0, \cdots, 0)}_{n}$ is a common n-tuple fixed point of $F, G_{2}, \cdots, G_{r}$.

Corollary 2.8. Let $(Y, d)$ be a Hausdorff left $K$-complete $T_{0}$-quasi-pseudometric space, $\phi: Y \rightarrow \mathbb{R}$ be a bounded from above function and $\preceq$ the preorder induced by $\phi$. Let $F: Y \times Y \rightarrow X$ and $G_{i}: Y \rightarrow Y ; i=$ $1,2, \cdots, r$ for $r>2$ be $N+1 d$-sequentially continuous mapping on $X$ such that the pairs $\left\{F, G_{r}\right\} ; r=2,3$ are weakly left-related. Moreover, we assume that $\left\{G_{r}, G_{r-1}, \cdots, G_{3}\right\}$ is a dual $r-2$-embedded chain. Then $F, G_{2}, \cdots, G_{r}$ have a common n-tuple fixed point in $Y$.

Corollary 2.9. Let $(Y, d)$ be a bicomplete $T_{0}$-quasi-pseudometric space, $\phi: Y \rightarrow \mathbb{R}$ be a bounded from above function and $\preceq$ the preorder induced by $\phi$. Let $F: Y \times Y \rightarrow X$ and $G_{i}: Y \rightarrow Y ; i=1,2, \cdots, r$ for $r>2$ be $N+1 d^{s}$-sequentially continuous mapping on $X$ such that the pairs $\left\{F, G_{r}\right\} ; r=2,3$ are weakly left-related. Moreover, we assume that $\left\{G_{r}, G_{r-1}, \cdots, G_{3}\right\}$ is an $r-2$-embedded chain. Then $F, G_{2}, \cdots, G_{r}$ have a common n-tuple fixed point in $Y$.

\section{Concluding Remark}

All the results given remain true when we replace accordingly the bicomplete quasi-pseudometric space $(X, d)$ by a left Smyth sequentially complete/left $K$-complete or a right Smyth sequentially complete/right $K$-complete space.

\section{References}

1. Ertürk, M, Karakaya, V; n-tuple fixed point theorems for contractive type mappings in partially ordered metric spaces. J. Inequal. Appl. 2013, 196 (2013).

2. Y. U. Gaba; "An order theoretic approach in fixed point theory." Mathematical Sciences (2014): 1-7. DOI 10.1007/s40096-014-0133-6.

3. V. Lakshmikantham and $\mathrm{Lj}$. B. Ciric; Coupled fixed point theorems for nonlinear contractions in partially ordered metric space, Nonlinear Anal. 70, no. 12, 4341-4349, 2009.

4. Y. U. Gaba; Startpoints and $(\alpha, \gamma)$-contractions in quasi-pseudometric spaces, Journal of Mathematics, Volume 2014 (2014), Article ID 709253, 8 pages http://dx.doi.org/10.1155/2014/709253. 